\title{
Mediastinal pancreatic pseudocyst
}

\author{
Mukesh Nasa, Narendra Choudhary, Gaurav Patil ${ }^{1}$, Rajesh Puri²
}

Consultant, ${ }^{1}$ DNB Fellow and ${ }^{2}$ Director, Institute of Digestive and Hepatobiliary Sciences, Medanta - The Medicity, Gurgaon, Haryana, India

$\begin{array}{ll}\text { Abstract } & \text { Pancreatic pseudocyst is a well-known complication of both acute and chronic pancreatitis. } \\ & \text { It is a collection of fluid due to acute or chronic inflammation of pancreas or from injury. } \\ \text { A pseudocyst with mediastinal extension is a rare entity. There are only few reports of } \\ \text { endoscopic transesophageal drainage of mediastinal pseudocysts. We present a case of } \\ \text { mediastinal pseudocyst in a 45-year-old male who presented with dysphagia, weight loss, fever, } \\ \text { and productive cough which was managed endoscopically by endoscopic ultrasound-guided } \\ \text { transesophageal aspiration. }\end{array}$

\section{Introduction}

Pseudocyst formation is a common complication of acute as well as chronic pancreatitis. Usually, pseudocysts are located within the pancreas or in the peri-pancreatic region. On rare occasions, mediastinal extension of pseudocyst can occur through anatomical openings of diaphragm. We report the case of a patient with a history of ethanol-induced acute pancreatitis presenting with difficulty in swallowing solid foods. Imaging revealed large cystic collection in the posterior mediastinum compressing esophagus.

\section{Case Report}

A 45-year-old male, known case of ethanol related pancreatitis 3 months back, now admitted with chief complaints of fever, productive cough for 5 days, and dysphagia since 1 month. At this admission, examination revealed left-sided crackles with normal air entry. His abdomen was soft with no organomegaly, palpable lump. His initial workup revealed polymorphonuclear leukocytosis with amylase 155 (0-110 U/L) and lipase 3001 (0-330 U/L). Chest X-ray

Address for correspondence:

Dr. Mukesh Nasa, Institute of Digestive and Hepatobiliary Sciences,

Medanta - The Medicity, Gurgaon, Haryana, India.

E-mail: mukeshnasa78@gmail.com

Access this article online

Website:

www.jdeonline.in

DOI:

10.4103/0976-5042.180095 showed left lower zone consolidation. He underwent computed tomography (CT) chest and abdomen which showed extensive peripancreatic inflammatory [Figures 1 and 2] change extending contiguously along the esophageal hiatus. There was a longitudinal fluid collection along the esophagus extending further superiorly into the neck in the retropharyngeal space with collapse consolidation in the lower lobe of left lung. Later, a magnetic resonance imaging (MRI) with magnetic resonance cholangiopancreatography (MRCP) was performed to delineate the pancreatic duct (PD) anatomy and the possible communication with pseudocyst. It showed a cystic retroesophageal collection [Figure 3] contiguously tracking from behind the cardiac end of the stomach, fluid pockets were noted in the distal body and tail of the pancreas and perisplenic region with no obvious communication between the PD and pseudocyst.

Clinically and radiologically, the patient has acute pancreatitis without organ failure, i.e., acute mild pancreatitis. His index event of pancreatitis ( 3 months back) was also mild.

The patient was taken for endoscopic retrograde cholangiopancreatography (ERCP). Pancreatogram showed no evidence of any PD leak. The ERCP and MRCP both did not demonstrate a PD communication.

This is an open access article distributed under the terms of the Creative Commons Attribution-NonCommercial-ShareAlike 3.0 License, which allows others to remix, tweak, and build upon the work non-commercially, as long as the author is credited and the new creations are licensed under the identical terms.

For reprints contact: reprints@medknow.com

How to cite this article: Nasa M, Choudhary N, Patil G, Puri R. Mediastinal pancreatic pseudocyst. J Dig Endosc 2016;7:20-3. 
As there was no prominent bulge in stomach or duodenum, transmural drainage of the pseudocyst was also not technically feasible. Patient was taken up for endoscopic ultrasound (EUS)-guided transesophageal aspiration of pseudocyst. There was bulge on the posterior esophageal wall with luminal narrowing, but echo-endoscope could be negotiated across.

There was minimal debris within the mediastinal fluid collection on EUS. Transesophageal aspiration of fluid collection was done with $19 \mathrm{G}$ needle [Figure 4]. About $600 \mathrm{ml}$ of turbid brown fluid was aspirated. The fluid analysis showed amylase of $10,900 \mathrm{IU} / \mathrm{ml}$. Postprocedure patient had immediate relief in the dysphagia. He was able to resume a normal diet within $24 \mathrm{~h}$. After 6 months of follow-up, patient is asymptomatic.

\section{Discussion}

An acute pancreatic fluid collection is a collection of pancreatic secretions and cellular debris. There is formation of a fibrous

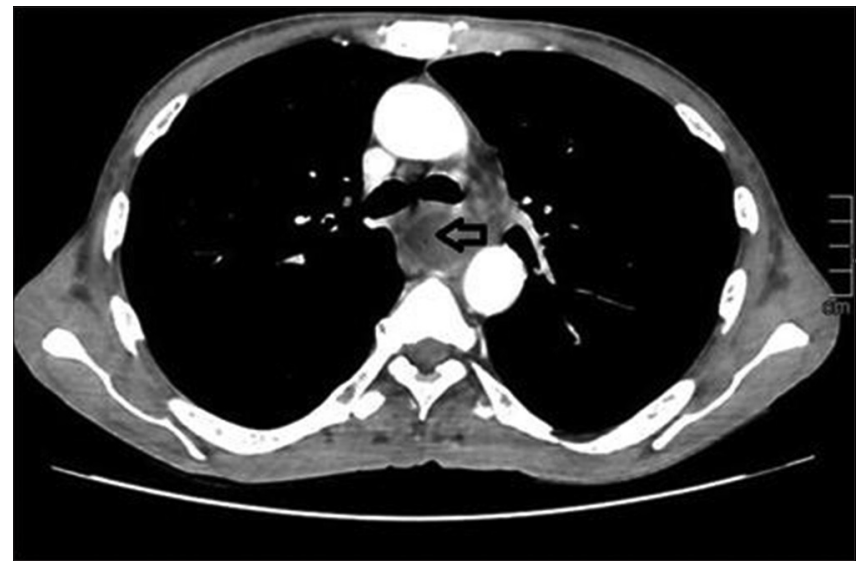

Figure 1: Contrast-enhanced computed tomography thorax showing posterior mediastinal pseudocyst compressing the esophagus

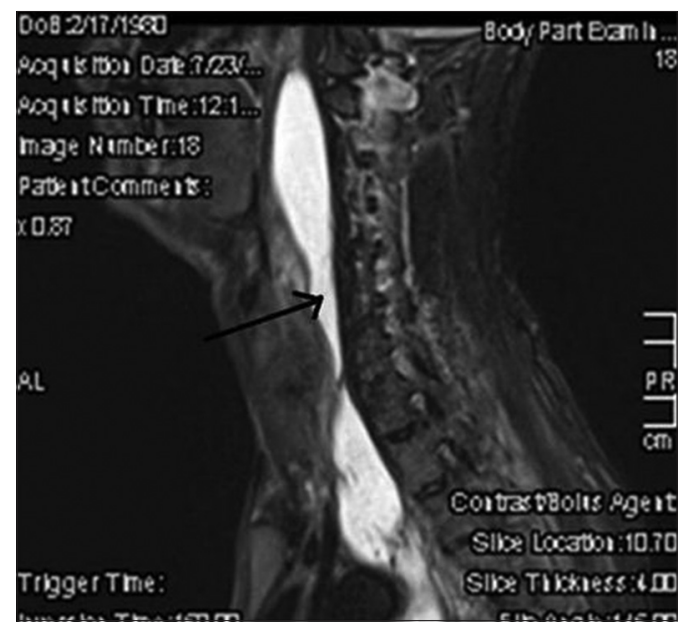

Figure 3: Magnetic resonance imaging (T2-weighted) image showing mediastinal pseudocyst extending into the neck capsule around the unresorbed collection of fluid after 4-6 weeks, thus forming a pseudocyst.

Pseudocysts are more frequently noted in chronic pancreatitis, complicating $20-25 \%$ of cases while it can complicate $7-15 \%$ of episodes of acute pancreatitis. ${ }^{[1]}$ These pseudocysts may resolve spontaneously in more than $50 \%$ of cases but about $5-40 \%$ can be associated with the complications. ${ }^{[2]}$ Among the different etiologies of pancreatitis which more frequently associated with pseudocyst formation are chronic alcoholism (75\%) and traumatic pancreatitis (13\%), carcinoma pancreas, gall stone pancreatitis, and idiopathic causes composing the remainders. ${ }^{[3]}$

We report an unusual case of mediastinal pseudocyst complicating alcoholic acute pancreatitis.

Our patient had presented 3 months earlier with mild alcoholic pancreatitis, and there was no pseudocyst or any evidence of chronic pancreatitis on the initial CT. The patient was

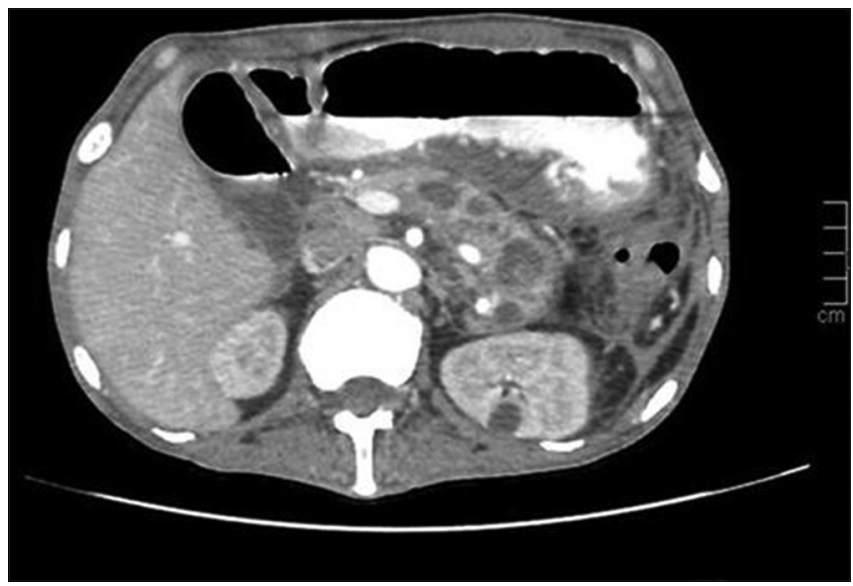

Figure 2: Contrast-enhanced computed tomography abdomen showing intrapancreatic collections and collection in the lesser-sac

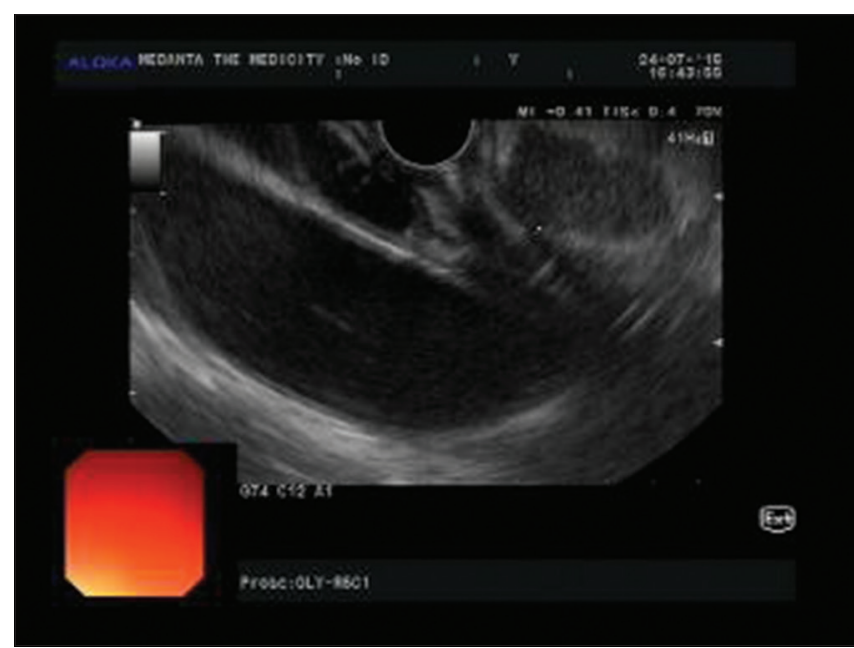

Figure 4: Endoscopic ultrasound image showing mediastinal pseudocyst with an-echoic contents 
discharged without any local or systemic complications of pancreatitis.

We assume pseudocyst formation occurred as a late complication of acute pancreatitis. As per revised Atlanta classification, it was moderate acute pancreatitis as there was local complication in form of pseudocyst formation. The ongoing slow accumulation of the fluid may have delayed the onset of symptoms, and hence, the patient never presented in the early stage of psuedocyst formation. In the present case, this pseudocyst extended into the posterior mediastinum up to skull base through esophageal hiatus causing esophageal compression resulting in dysphagia with subsequent aspiration pneumonitis.

The extra-abdominal extension of a pancreatic pseudocyst is rare. Mediastinal pseudocyst occurs as a result of tracking of the fluid in the fascial planes which offer the path of least resistance. Posterior mediastinal pancreatic pseudocysts occur as a result of tracking of pseudocyst fluid through the esophageal and aortic hiatus; therefore, the posterior mediastinum is the most common location of the mediastinal pseudocysts. ${ }^{[4]}$ Extension through foramen of Morgagni is less common while mid-mediastinal extension has also been reported rarely due to direct erosion through the diaphragm. Complications due to mediastinal pancreatic pseudocyst can occur due to invasion, compression of the mediastinal structures, or rupture of pseudocyst into surrounding structures. ${ }^{[5]}$ Mediastinal pseudocysts may rupture into the pleural cavity, thus producing pancreatic pleural effusion. The pressure of enlarging pseudocyst on cardiac chambers can cause cardiac filling pressure causing congestive cardiac failure ${ }^{[6]}$ Rare case of cardiac tamponade due to rupture of mediastinal pseudocyst into the pericardial sac has been reported. ${ }^{[7]}$

Cross-sectional imaging helps to diagnose the mediastinal pseudocyst. CT scan demonstrates the location and its relation to the surrounding structures while MRI can show the fistulous communication with the pancreas. Spontaneous resolution of the mediastinal pseudocysts is rare. Somatostatin or its analogs decrease pancreatic juice production and may help in decreasing the mediastinal pseudocysts but require prolonged therapy. ${ }^{[8]}$ Management of the mediastinal pseudocysts depends on the location, its relation to the surrounding structures, symptoms, presence or absence of communication with the $\mathrm{PD}$, and the expertise available.

Once communication with the PD is established on cross-sectional imaging, ERCP is a useful modality for transpapillary or transgastric drainage of the pseudocyst.

PD leak on ERCP is seen in $46-78 \%$ cases only. ${ }^{[9]}$

Transcutaneous drainage of the abdominal pseudocyst has risk of the infection, bleeding, and recurrence. Endoscopic drainage procedures has recurrence of $>5 \%$ and complication rate of
$10 \%$ with better tolerability while transcutaneous drainage has recurrence rate of $20 \%$ and risk of infection of about $12 \%$. $^{[10]}$ Further repeat EUS after resolution of medistinal pseudocysts has shown significant periesophageal fibrosis and rarely a stricture needing endoscopic Bougie dilatation. ${ }^{[11]}$

The pseudocyst with bulge in the stomach or duodenum can easily be drained endoscopically. EUS-guided pseudocyst drainage is used if the transmural bulge is not prominent. EUS-guided transgastric drainage with placement of double pigtail stent has been shown to resolve the medistinal pseudocyst. ${ }^{[12]}$ In our case, there was no stomach or duodenal bulge; hence, this was avoided. EUS demonstrates the thickness and any vessel in the intervening wall.

Another modality of drainage would have been a cystoesophagostomy and placement of a fully covered metal stent with anchor flanges to oppose the nonadherent lumen. ${ }^{[13]}$ In a case report by Sugimoto, EUS-guided trans-gastric drainage of mediastinal pseudocyst with placement of double-pigtail plastic stent has been reported. ${ }^{[12]}$ In our patient, there was no bulge within the gastric lumen and patient reported immediate relief of dysphagia after EUS-guided transesophageal-aspiration.

\section{Conclusions}

A mediastinal pseudocyst is rare complication of acute pancreatitis. In a patient with the clinical history of pancreatitis presenting with dysphagia, dyspnea, or atypical chest pain, mediastinal pancreatic pseudocyst should be suspected. The finding of a thin-walled low-attenuation cystic mass in the posterior or middle mediastinum in continuity with pancreas and an elevated amylase level on analysis of the cystic content establishes the definitive diagnosis. This case report has shown that EUS-guided drainage is a useful modality for patients having a mediastinal pseudocyst.

\section{Financial support and sponsorship}

Nil.

\section{Conflicts of interest}

There are no conflicts of interest.

\section{References}

1. Vosoghi M, Sial S, Garrett B, Feng J, Lee T, Stabile BE, et al. EUS-guided pancreatic pseudocyst drainage: Review and experience at Harbor-UCLA medical center. MedGenMed 2002;4:2.

2. Vitas GJ, Sarr MG. Selected management of pancreatic pseudocysts: Operative versus expectant management. Surgery 1992;111:123-30.

3. Balthazar EJ. Pancreatitis. In: Gore RM, Levine MS, editors. Text Book of Gastrointestinal Radiology. $2^{\text {nd }}$ ed. Philadelphia: WB Saunders; 2002. p. $1785-7$.

4. Jeung MY, Gasser B, Gangi A, Bogorin A, Charneau D, Wihlm JM, et al. Imaging of cystic masses of the mediastinum. Radiographics 2002;22:S79-93. 
5. Komtong S, Chanatrirattanapan R, Kongkam P, Rerknimitr R, Kullavanijaya P. Mediastinal pseudocyst with pericardial effusion and dysphagia treated by endoscopic drainage. JOP 2006;7:405-10.

6. Lee FY, Wang YT, Poh SC. Congestive heart failure due to a pancreatic pseudocyst. Cleve Clin J Med 1994;61:141-3.

7. Tan MH, Kirk G, Archibold P, Kennedy P, Regan MC. Cardiac compromise due to a pancreatic mediastinal pseudocyst. Eur J Gastroenterol Hepatol 2002;14:1279-82.

8. Yasuda H, Ino Y, Igarashi $\mathrm{H}$, Arita $\mathrm{Y}$, Nakamuta $\mathrm{M}$, Sumii T, et al. A case of pancreatic pleural effusion and mediastinal pancreatic pseudocyst: Management by a somatostatin analogue octreotide. Pancreas 1999;19:410-2.

9. Ranjan P, Bansal R, Sachdeva M, Kumar M. Pancreaticopleural fistula: Report of two cases and review of literature. J Dig Endosc 2013;4:123-7.
10. Bardia A, Stoikes N, Wilkinson NW. Mediastinal pancreatic pseudocyst with acute airway obstruction. J Gastrointest Surg 2006;10:146-50.

11. Bhasin DK, Rana SS, Rao C, Gupta R, Kang M, Sinha SK, et al. Clinical presentation, radiological features, and endoscopic management of mediastinal pseudocysts: Experience of a decade. Gastrointest Endosc 2012;76:1056-60.

12. Sugimoto S, Yamagishi Y, Higuchi H, Kanai T. Endoscopic ultrasound-guided drainage for a mediastinal pancreatic pseudocyst. Intern Med 2014;53:2651-2.

13. Gornals JB, Loras C, Mast R, Botargues JM, Busquets J, Castellote J. Endoscopic ultrasound-guided transesophageal drainage of a mediastinal pancreatic pseudocyst using a novel lumen-apposing metal stent. Endoscopy 2012;44 Suppl 2:E211-2.

\section{Author Help: Reference checking facility}

The manuscript system (www.journalonweb.com) allows the authors to check and verify the accuracy and style of references. The tool checks the references with PubMed as per a predefined style. Authors are encouraged to use this facility, before submitting articles to the journal.

- The style as well as bibliographic elements should be $100 \%$ accurate, to help get the references verified from the system. Even a single spelling error or addition of issue number/month of publication will lead to an error when verifying the reference.

- Example of a correct style

Sheahan P, O'leary G, Lee G, Fitzgibbon J. Cystic cervical metastases: Incidence and diagnosis using fine needle aspiration biopsy. Otolaryngol Head Neck Surg 2002;127:294-8.

- Only the references from journals indexed in PubMed will be checked.

- Enter each reference in new line, without a serial number.

- Add up to a maximum of 15 references at a time.

- If the reference is correct for its bibliographic elements and punctuations, it will be shown as CORRECT and a link to the correct article in PubMed will be given.

- If any of the bibliographic elements are missing, incorrect or extra (such as issue number), it will be shown as INCORRECT and link to possible articles in PubMed will be given. 